\title{
The dual decomposition of aggregation functions and its application in welfare economics
}

\author{
José Luis García-Lapresta \\ PRESAD Research Group, IMUVA, Departamento de Economía Aplicada, Universidad de \\ Valladolid, Avenida Valle de Esgueva 6, 47011 Valladolid, Spain \\ Ricardo Alberto Marques Pereira \\ Dipartimento di Economia e Management, Università degli Studi di Trento, Via Inama 5, \\ TN 38122, Trento, Italy
}

\begin{abstract}
In this paper, we review the role of self-duality in the theory of aggregation functions, the dual decomposition of aggregation functions into a self-dual core and an anti-self-dual remainder, and some applications to welfare, inequality, and poverty measures.
\end{abstract}

Keywords: aggregation functions; self-duality; dual decomposition; inequality; welfare; poverty

\section{Introduction}

In the context of aggregation functions, self-duality is an important property (see Beliakov et al. [3] and Grabisch et al. [16]). Self-dual aggregation functions satisfy $A(\mathbf{1}-\boldsymbol{x})=1-A(\boldsymbol{x})$ for every $\boldsymbol{x} \in[0,1]^{n}$. In other words, the aggregate value of the transformed inputs coincides with the transformed aggregate value of the original inputs. This means that the aggregation function is unbiased relatively to the higher or lower value of its inputs.

In the aggregation of reciprocal preference relations, for instance, self-duality ensures the reciprocity of the aggregate preference relation (see García-Lapresta and Llamazares [12]).

Silvert [24] introduced symmetric sums, a class of self-dual aggregation functions with two variables, within the context of his characterization of self-duality (see also Dubois and Prade [8] and Calvo et al. [6, p. 32]).

García-Lapresta and Marques Pereira $[13,14]$ proposed a method that associates a self-dual aggregation function to any aggregation function. This method

Email addresses: lapresta@eco.uva.es (José Luis García-Lapresta), ricalb.marper@unitn.it (Ricardo Alberto Marques Pereira)

Preprint submitted to Fuzzy Sets and Systems 
improves the one given by Silvert [24] in a number of ways (see García-Lapresta and Marques Pereira [14, Sect. 4]).

Maes et al. [20] provide a characterization of self-dual aggregation functions which generalizes those given by Silvert [24] and García-Lapresta and Marques Pereira [14]. In turn, Maes and De Baets [19] merge self-dual and commutative binary aggregation functions in a single functional equation.

The paper is organized as follows. Section 2 reviews basic notions regarding aggregation functions and their dual decomposition, with a particular focus on exponential means and OWA functions. Section 3 discusses some applications of the dual decomposition to welfare economics, and Section 4 contains some concluding remarks.

\section{Aggregation functions}

We now present notation and basic definitions regarding aggregation functions on $[0,1]^{n}$, with $n \in \mathbb{N}$ and $n \geq 2$ throughout the text. For further details the interested reader is referred to Fodor and Roubens [10], Calvo et al. [6], Beliakov et al. [3], García-Lapresta and Marques Pereira [14] and Grabisch et al. $[16]$.

Vectors in $[0,1]^{n}$ are denoted as $\boldsymbol{x}=\left(x_{1}, \ldots, x_{n}\right), \quad \mathbf{0}=(0, \ldots, 0), \mathbf{1}=$ $(1, \ldots, 1)$. Accordingly, for every $x \in[0,1]$, we have $x \cdot \mathbf{1}=(x, \ldots, x)$. Given $\boldsymbol{x}, \boldsymbol{y} \in[0,1]^{n}$, by $\boldsymbol{x} \geq \boldsymbol{y}$ we mean $x_{i} \geq y_{i}$ for every $i \in\{1, \ldots, n\}$, and by $\boldsymbol{x}>\boldsymbol{y}$ we mean $\boldsymbol{x} \geq \boldsymbol{y}$ and $\boldsymbol{x} \neq \boldsymbol{y}$. Given $\boldsymbol{x} \in[0,1]^{n}$, the increasing and decreasing reorderings of the coordinates of $\boldsymbol{x}$ are indicated as $x_{(1)} \leq \cdots \leq x_{(n)}$ and $x_{[1]} \geq \cdots \geq x_{[n]}$, respectively. In particular, $x_{(1)}=\min \left\{x_{1}, \ldots, x_{n}\right\}=$ $x_{[n]}$ and $x_{(n)}=\max \left\{x_{1}, \ldots, x_{n}\right\}=x_{[1]}$. Clearly, $x_{[k]}=x_{(n-k+1)}$ for every $k \in\{1, \ldots, n\}$. In general, given a permutation $\sigma$ on $\{1, \ldots, n\}$, we denote $\boldsymbol{x}_{\sigma}=\left(x_{\sigma(1)}, \ldots, x_{\sigma(n)}\right)$. The arithmetic mean of $\boldsymbol{x}$ is denoted by $\mu(\boldsymbol{x})$.

Definition 1. Let $A:[0,1]^{n} \longrightarrow \mathbb{R}$ be a function.

1. $A$ is idempotent if for every $x \in[0,1]$ it holds that $A(x \cdot \mathbf{1})=x$.

2. $A$ is symmetric if for every permutation $\sigma$ on $\{1, \ldots, n\}$ and every $\boldsymbol{x} \in$ $[0,1]^{n}$ it holds that $A\left(\boldsymbol{x}_{\sigma}\right)=A(\boldsymbol{x})$.

3. $A$ is monotonic if for all $\boldsymbol{x}, \boldsymbol{y} \in[0,1]^{n}$ it holds that $\boldsymbol{x} \geq \boldsymbol{y} \Rightarrow A(\boldsymbol{x}) \geq$ $A(\boldsymbol{y})$.

4. A is strictly monotonic if for all $\boldsymbol{x}, \boldsymbol{y} \in[0,1]^{n}$ it holds that $\boldsymbol{x}>\boldsymbol{y} \Rightarrow$ $A(\boldsymbol{x})>A(\boldsymbol{y})$.

5. A is compensative (or internal) if for every $\boldsymbol{x} \in[0,1]^{n}$ it holds that $x_{(1)} \leq A(\boldsymbol{x}) \leq x_{(n)}$.

6. A is self-dual if for every $\boldsymbol{x} \in[0,1]^{n}$ it holds that $A(\mathbf{1}-\boldsymbol{x})=1-A(\boldsymbol{x})$.

7. $A$ is anti-self-dual if for every $\boldsymbol{x} \in[0,1]^{n}$ it holds that $A(\mathbf{1}-\boldsymbol{x})=A(\boldsymbol{x})$.

8. A is invariant for translations if for every $\boldsymbol{x} \in[0,1]^{n}$ it holds that $A(\boldsymbol{x}+$ $t \cdot \mathbf{1})=A(\boldsymbol{x})$ for every $t \in \mathbb{R}$ such that $\boldsymbol{x}+t \cdot \mathbf{1} \in[0,1]^{n}$.

9. A is stable for translations (or shift-invariant) if for every $\boldsymbol{x} \in[0,1]^{n}$ it holds that $A(\boldsymbol{x}+t \cdot \mathbf{1})=A(\boldsymbol{x})+t$ for every $t \in \mathbb{R}$ such that $\boldsymbol{x}+t \cdot \mathbf{1} \in[0,1]^{n}$. 
Definition 2. Let $\left(A^{(k)}\right)_{k \in \mathbb{N}}$ be a sequence of functions, with $A^{(k)}:[0,1]^{k} \longrightarrow$ $\mathbb{R}$ and $A^{(1)}(x)=x$ for every $x \in[0,1] .\left(A^{(k)}\right)_{k \in \mathbb{N}}$ is invariant for replications (or strongly idempotent) if for all $\boldsymbol{x} \in[0,1]^{n}$ and any number of replications $m \in \mathbb{N}$ of $\boldsymbol{x}$ it holds that

$$
A^{(m n)}(\overbrace{\boldsymbol{x}, \ldots, \boldsymbol{x}}^{m})=A^{(n)}(\boldsymbol{x}) .
$$

Definition 3. Consider the binary relation $\succcurlyeq$ on $[0,1]^{n}$, defined as

$$
\boldsymbol{x} \succcurlyeq \boldsymbol{y} \Leftrightarrow \sum_{i=1}^{n} x_{i}=\sum_{i=1}^{n} y_{i} \text { and } \sum_{i=1}^{k} x_{(i)} \leq \sum_{i=1}^{k} y_{(i)},
$$

for every $k \in\{1, \ldots, n-1\}$.

1. A function $A:[0,1]^{n} \longrightarrow[0,1]$ is S-convex if for all $\boldsymbol{x}, \boldsymbol{y} \in[0,1]^{n}$ :

$$
\boldsymbol{x} \succcurlyeq \boldsymbol{y} \Rightarrow A(\boldsymbol{x}) \geq A(\boldsymbol{y})
$$

2. A function $A:[0,1]^{n} \longrightarrow[0,1]$ is strictly S-convex if for all $\boldsymbol{x}, \boldsymbol{y} \in[0,1]^{n}$ :

$$
\boldsymbol{x} \succ \boldsymbol{y} \Rightarrow A(\boldsymbol{x})>A(\boldsymbol{y}),
$$

where $\boldsymbol{x} \succ \boldsymbol{y}$ means $\boldsymbol{x} \succcurlyeq \boldsymbol{y}$ and $\boldsymbol{x} \neq \boldsymbol{y}$.

Definition 4. A function $A:[0,1]^{n} \longrightarrow[0,1]$ is called an $n$-ary aggregation function if it is monotonic and satisfies $A(\mathbf{1})=1$ and $A(\mathbf{0})=0$. An aggregation function is said to be strict if it is strictly monotonic.

For the sake of simplicity, the $n$-arity is omitted whenever it is clear from the context. Every idempotent aggregation function is compensative, and vice versa.

\subsection{Dual decomposition of aggregation functions}

We now briefly recall the so-called dual decomposition of an aggregation function into its self-dual core and associated anti-self-dual remainder, due to García-Lapresta and Marques Pereira [14]. First we introduce the concepts of self-dual core and anti-self-dual remainder of an aggregation function, establishing which properties are inherited in each case from the original aggregation function. Particular emphasis is given to the properties of stability for translations (self-dual core) and invariance for translations (anti-self-dual remainder).

Definition 5. Let $A:[0,1]^{n} \longrightarrow[0,1]$ be an aggregation function. The aggregation function $A^{*}:[0,1]^{n} \longrightarrow[0,1]$ defined as

$$
A^{*}(\boldsymbol{x})=1-A(\mathbf{1}-\boldsymbol{x})
$$

is known as the dual of the aggregation function $A$.

Clearly, $\left(A^{*}\right)^{*}=A$. Thus, an aggregation function $A$ is self-dual if and only if $A^{*}=A$. Moreover, the dual $A^{*}$ inherits from the aggregation function $A$ the properties of continuity, idempotency (hence, compensativeness), symmetry, strict monotonicity, self-duality, stability for translations and invariance for replications, whenever $A$ has these properties. 


\subsubsection{The self-dual core of an aggregation function}

Aggregation functions are not in general self-dual. However, a self-dual aggregation function can be associated with any aggregation function in a simple manner.

Definition 6. Let $A:[0,1]^{n} \longrightarrow[0,1]$ be an aggregation function. The function $\widehat{A}:[0,1]^{n} \longrightarrow[0,1]$ defined as

$$
\widehat{A}(\boldsymbol{x})=\frac{A(\boldsymbol{x})+A^{*}(\boldsymbol{x})}{2}=\frac{A(\boldsymbol{x})-A(\mathbf{1}-\boldsymbol{x})+1}{2}
$$

is called the core of the aggregation function $A$.

Since $\widehat{A}$ is self-dual, we say that $\widehat{A}$ is the self-dual core of the aggregation function $A$. Notice that $\widehat{A}$ is clearly an aggregation function.

The following results ${ }^{1}$ can be found in García-Lapresta and Marques Pereira [14].

Proposition 1. An aggregation function $A:[0,1]^{n} \longrightarrow[0,1]$ is self-dual if and only if $\widehat{A}(\boldsymbol{x})=A(\boldsymbol{x})$ for every $\boldsymbol{x} \in[0,1]^{n}$.

Proposition 2. The self-dual core $\widehat{A}$ inherits from the aggregation function $A$ the properties of continuity, idempotency (hence, compensativeness), symmetry, strict monotonicity, stability for translations, and invariance for replications, whenever A has these properties.

\subsubsection{The anti-self-dual remainder of an aggregation function}

We now introduce the anti-self-dual remainder $\widetilde{A}$, which is simply the difference between the original aggregation function $A$ and its self-dual core $\widehat{A}$.

Definition 7. Let $A:[0,1]^{n} \longrightarrow[0,1]$ be an aggregation function. The function $\widetilde{A}:[0,1]^{n} \longrightarrow \mathbb{R}$ defined as $\widetilde{A}(\boldsymbol{x})=A(\boldsymbol{x})-\widehat{A}(\boldsymbol{x})$, that is,

$$
\widetilde{A}(\boldsymbol{x})=\frac{A(\boldsymbol{x})-A^{*}(\boldsymbol{x})}{2}=\frac{A(\boldsymbol{x})+A(\mathbf{1}-\boldsymbol{x})-1}{2},
$$

is called the remainder of the aggregation function $A$.

Since $\widetilde{A}$ is anti-self-dual, we say that $\widetilde{A}$ is the anti-self-dual remainder of the aggregation function $A$. Clearly, $\widetilde{A}$ is not an aggregation function. In particular, $\widetilde{A}(\mathbf{0})=\widetilde{A}(\mathbf{1})=0$ violates idempotency and implies that $\widetilde{A}$ is either non monotonic or everywhere null.

The following results ${ }^{2}$ can be found in García-Lapresta and Marques Pereira [14].

\footnotetext{
${ }^{1}$ Excepting that invariance for replications is inherited by the core (the proof is immediate).

${ }^{2}$ Excepting that invariance for replications is inherited by the remainder (the proof is immediate).
} 
Proposition 3. An aggregation function $A:[0,1]^{n} \longrightarrow[0,1]$ is self-dual if and only if $\widetilde{A}(\boldsymbol{x})=0$ for every $\boldsymbol{x} \in[0,1]^{n}$.

Proposition 4. The anti-self-dual remainder $\widetilde{A}$ inherits from the aggregation function $A$ the properties of continuity, symmetry, and invariance for replications, whenever A has these properties.

Summarizing, every aggregation function $A$ decomposes additively $A=$ $\widehat{A}+\widetilde{A}$ in two components: the self-dual core $\widehat{A}$ and the anti-self-dual remainder $\widetilde{A}$, where only $\widehat{A}$ is an aggregation function.

The following result concerns two more properties of the anti-self-dual remainder based directly on the definition $\widetilde{A}=A-\widehat{A}$ and the corresponding properties of the self-dual core (see García-Lapresta and Marques Pereira [14]).

Proposition 5. Let $A:[0,1]^{n} \longrightarrow[0,1]$ be an aggregation function.

1. If $A$ is idempotent, then $\widetilde{A}(x \cdot \mathbf{1})=0$ for every $x \in[0,1]$.

2. If $A$ is stable for translations, then $\widetilde{A}$ is invariant for translations.

These properties of the anti-self-dual remainder are suggestive. The first statement establishes that anti-self-dual remainders of idempotent aggregation functions are null on the main diagonal. The second statement applies to the subclass of stable aggregation functions. In such case, self-dual cores are stable and therefore anti-self-dual remainders are invariant for translations. In other words, if the aggregation function $A$ is stable for translations, the value $\widetilde{A}(\boldsymbol{x})$ does not depend on the average value of the $\boldsymbol{x}$ coordinates, but only on their numerical deviations from that average value. These properties of the anti-selfdual remainder $\widetilde{A}$ suggest that it may give some indication on the dispersion of the $\boldsymbol{x}$ coordinates.

In Maes et al. [20], the authors propose a generalization of the dual decomposition framework introduced in García-Lapresta and Marques Pereira [14], based on a family of binary aggregation functions satisfying a form of twisted self-duality condition. Each binary aggregation function in that family corresponds to a particular way of combining an aggregation function $A$ with its dual $A^{*}$ for the construction of the self-dual core $\widehat{A}$. As particular cases of the general framework proposed in Maes et al. [20], one obtains García-Lapresta and Marques Pereira's construction, based on the arithmetic mean, and Silvert's construction, based on the symmetric sums formula (see Silvert [24]). However, the dual decomposition framework introduced in García-Lapresta and Marques Pereira [14] remains the only one which preserves stability under translations.

\subsection{Dual decomposition of exponential means}

Quasiarithmetic means are the only aggregation functions satisfying continuity, idempotency, symmetry, strict monotonicity and decomposability (see Kolmogoroff [18], Nagumo [22] and Fodor and Roubens [10, pp. 112-114]).

Exponential means are the only quasiarithmetic means satisfying stability for translations. 
Given $\alpha \neq 0$, the exponential mean $A_{\alpha}$ is the aggregation function defined as

$$
A_{\alpha}(\boldsymbol{x})=\frac{1}{\alpha} \ln \frac{e^{\alpha x_{1}}+\cdots+e^{\alpha x_{n}}}{n} .
$$

We now describe the dual decomposition of exponential means (see GarcíaLapresta and Marques Pereira [14, Sect. 6] for more details).

Given $\alpha \neq 0$, the self-dual core of $A_{\alpha}$ is the aggregation function $\widehat{A}_{\alpha}$ defined as

$$
\widehat{A}_{\alpha}(\boldsymbol{x})=\frac{1}{2 \alpha} \ln \frac{e^{\alpha x_{1}}+\cdots+e^{\alpha x_{n}}}{e^{-\alpha x_{1}}+\cdots+e^{-\alpha x_{n}}} .
$$

For every $\alpha \neq 0, \widehat{A}_{\alpha}$ is continuous, idempotent, symmetric, strictly monotonic, compensative, stable for translations, self-dual and invariant for replications.

Given $\alpha \neq 0$, the anti-self-dual remainder of $A_{\alpha}$ is the mapping $\widetilde{A}_{\alpha}$ defined as

$$
\widetilde{A}_{\alpha}(\boldsymbol{x})=\frac{1}{2 \alpha} \ln \frac{\left(e^{\alpha x_{1}}+\cdots+e^{\alpha x_{n}}\right)\left(e^{-\alpha x_{1}}+\cdots+e^{-\alpha x_{n}}\right)}{n^{2}} .
$$

For every $\alpha \neq 0, \widetilde{A}_{\alpha}(\boldsymbol{x})=0$ if and only if $x_{1}=\cdots=x_{n}$. Moreover, $\widetilde{A}_{\alpha}$ is continuous, symmetric, anti-self-dual, invariant for translations and invariant for replications.

The previous decomposition will be used in Subsection 3.1.

\subsection{Dual decomposition of OWA functions}

OWA functions, introduced by Yager [25], are aggregation functions satisfying continuity, idempotency (hence, compensativeness), symmetry and stability for translations (see Fodor et al. [9]).

Given a weighting vector $\boldsymbol{w}=\left(w_{1}, \ldots, w_{n}\right) \in[0,1]^{n}$ satisfying $\sum_{i=1}^{n} w_{i}=1$, the $O W A$ function associated with $\boldsymbol{w}$ is the aggregation function $A_{\boldsymbol{w}}:[0,1]^{n} \longrightarrow$ $[0,1]$ defined as

$$
A_{\boldsymbol{w}}(\boldsymbol{x})=\sum_{i=1}^{n} w_{i} x_{[i]} .
$$

Notice that an OWA function $A_{\boldsymbol{w}}$ is self-dual if and only if $w_{n+1-i}=w_{i}$ for every $i \in\{1, \ldots, n\}$.

We now describe the dual decomposition of OWA functions (see GarcíaLapresta and Marques Pereira [14, Sect. 5] for more details).

The self-dual core $\widehat{A}_{\boldsymbol{w}}$ and the anti-self-dual remainder $\widetilde{A}_{\boldsymbol{w}}$ of an OWA function $A_{w}$ can be written as

$$
\widehat{A}_{\boldsymbol{w}}(\boldsymbol{x})=\sum_{i=1}^{n} \frac{w_{i}+w_{n+1-i}}{2} x_{[i]} \quad \text { and } \quad \widetilde{A}_{\boldsymbol{w}}(\boldsymbol{x})=\sum_{i=1}^{n} \frac{w_{i}-w_{n+1-i}}{2} x_{[i]} .
$$

The dual decomposition will be used in Subsections 3.1 and 3.2. 


\section{Some applications to welfare economics}

In this section we briefly describe some applications of self-duality and the dual decomposition of exponential means and OWA functions to the field of welfare economics: in the measurement of poverty, inequality, and welfare. Further details can be found in García-Lapresta et al. [11] and Aristondo et al. [1, 2].

We consider a population consisting of $n$ individuals, with $n \geq 2$. An income distribution is represented by a vector $\boldsymbol{x}=\left(x_{1}, \ldots, x_{n}\right) \in[0,1]^{n}$, where $x_{i} \in[0,1]$ represents the income of individual $i \in\{1, \ldots, n\}$. The unit interval is the natural domain in dual decomposition framework, although most of the constructions and results presented extend straightforwardly to the traditional income domain $[0, \infty)$ (see Aristondo et al. [2, Remarks 2-3]).

\subsection{Applications to the measurement of poverty}

According to Sen [23], a poverty measure consists essentially of an aggregation procedure within the poor sector of the population. The identification of the poor individuals requires the specification of a poverty line $z \in(0,1)$ which represents the necessary income to maintain a minimum level of living. Given an income distribution $\boldsymbol{x}$, an individual is considered to be poor if his/her income is below the poverty line, $x<z$. Otherwise he/she is non-poor.

The set of poor individuals in the population is denoted by

$$
Q(\boldsymbol{x}, z)=\left\{i \in\{1, \ldots, n\} \mid x_{i}<z\right\},
$$

and $q(\boldsymbol{x}, z)$ denotes the number of the poor, $q(\boldsymbol{x}, z)=\# Q(\boldsymbol{x}, z)$.

Once the poor have been identified, an aggregation procedure determines the extent of poverty in the population.

The first poverty measure introduced in the literature is the headcount ratio, $H:[0,1]^{n} \times(0,1) \longrightarrow[0,1]$, defined as

$$
H(\boldsymbol{x}, z)=\frac{q(\boldsymbol{x}, z)}{n},
$$

which measures the percentage of poor individuals in the population.

For all $\boldsymbol{x} \in[0,1]^{n}$ and $z \in(0,1)$, the normalized gap of individual $i$ is defined as

$$
g_{i}=\max \left\{\frac{z-x_{i}}{z}, 0\right\} .
$$

Notice that $g_{i} \in[0,1], g_{i}=0 \Leftrightarrow x_{i} \geq z$, and $g_{i}=1 \Leftrightarrow x_{i}=0$.

At this point it is useful to introduce a special notation for the incomes and normalized gaps of the poor individuals in the population: $\boldsymbol{x}_{p}=\left(x_{(1)}, \ldots, x_{(q)}\right)$ with $x_{(1)} \leq \cdots \leq x_{(q)}<z$, and $\boldsymbol{g}_{p}=\left(g_{[1]}, \ldots, g_{[q]}\right)$ with $g_{[1]} \geq \cdots \geq g_{[q]}>0$, and $g_{[i]}=\left(z-x_{(i)}\right) / z$ for $i=1, \ldots, q$.

In García-Lapresta et al. [11] the following poverty measure has been proposed. 
Definition 8. Given $\alpha>0$, the poverty measure associated with $A_{\alpha}$ is the function $P_{\alpha}:[0,1]^{n} \times(0,1) \longrightarrow[0,1]^{n}$ defined by

$$
P_{\alpha}(\boldsymbol{x}, z)= \begin{cases}H(\boldsymbol{x}, z) \cdot A_{\alpha}\left(\boldsymbol{g}_{p}\right)=\frac{q}{n} \cdot \frac{1}{\alpha} \ln \frac{e^{\alpha g_{[1]}}+\cdots+e^{\alpha g_{[q]}}}{q}, & \text { if } q \neq 0, \\ 0, & \text { if } q=0,\end{cases}
$$

where $q=q(\boldsymbol{x}, z)$ and $g_{[1]}, \ldots, g_{[q]}>0$ are the normalized gaps of the poor individuals in the population.

For every $\alpha>0, P_{\alpha}$ satisfies interesting properties (see García-Lapresta et al. [11, Prop. 17]): Poverty Focus (poverty should not depend on the non-poor incomes), Poverty Monotonicity (poverty should increase if a poor income decreases), Transfer Sensitivity (greater weight should be placed on the poorer incomes and poverty should decrease if inequality among the poor decreases), Normalization (if all the individuals are non-poor, then the society deprivation level is equal to 0), Poverty Symmetry (no other characteristic apart from the income deprivation matters in defining a poverty index), and Replication Invariance (if the population is replicated, then poverty should not change; this allows comparing populations of different sizes).

Every poverty measure should be expressed as a function of three poverty indicators: incidence, intensity and inequality of the poverty (see Sen [23] and Jenkins and Lambert [17]).

In our case, for every $\alpha>0$, the poverty measure $P_{\alpha}$ associated with $A_{\alpha}$ can be decomposed in the following way:

$$
P_{\alpha}(\boldsymbol{x}, z)= \begin{cases}H(\boldsymbol{x}, z) \cdot\left(\widehat{A}_{\alpha}\left(\boldsymbol{g}_{p}\right)+\widetilde{A}_{\alpha}\left(\boldsymbol{g}_{p}\right)\right), & \text { if } q \neq 0, \\ 0, & \text { if } q=0,\end{cases}
$$

where $q=q(\boldsymbol{x}, z)$ and $g_{[1]}, \ldots, g_{[q]}>0$ are the normalized gaps of the poor individuals in the population.

Thus, the poverty measure $P_{\alpha}$ is clearly expressed through the three above mentioned poverty indicators: incidence, intensity and inequality, by means of $H, \widehat{A}_{\alpha}$ and $\widetilde{A}_{\alpha}$, respectively.

We now discuss an application of the dual decomposition in the context of a well-known poverty measure proposed by Sen, following Aristondo et al. [1].

An inequality measure is a summary statistics of income dispersion. In turn, welfare measures depend on the mean income and on the income inequality.

When inequality needs to be assessed, one may focus either on achievements or on shortfalls. However, the respective inequality rankings do not in general coincide.

In the framework proposed by Sen, $S:[0,1]^{n} \times(0,1) \longrightarrow[0,1]$, the poverty measure is defined as

$$
S(\boldsymbol{x}, z)=\frac{1}{q n} \sum_{i=1}^{q}(2(q-i)+1) g_{[i]},
$$


and the inequality among the poor is captured by the absolute Gini index, which can be expressed in terms of achievements $\boldsymbol{x}_{p}=\left(x_{(1)}, \ldots, x_{(q)}\right)$ :

$$
G_{A}\left(\boldsymbol{x}_{p}\right)=\mu\left(\boldsymbol{x}_{p}\right)-\sum_{i=1}^{q} \frac{2(q-i)+1}{q^{2}} x_{(i)} \text {. }
$$

or, alternatively, in terms of shortfalls $\boldsymbol{g}_{p}=\left(g_{[1]}, \ldots, g_{[q]}\right)$ :

$$
G_{A}\left(\boldsymbol{g}_{p}\right)=\mu\left(\boldsymbol{g}_{p}\right)-\sum_{i=1}^{q} \frac{2 i-1}{q^{2}} g_{[i]}
$$

In order to understand the actual relation between the two inequality assessments in terms of the absolute Gini index, we consider the dual decomposition of OWA functions discussed in Subsection 2.3.

In Aristondo et al. [1] the Sen poverty measure is rewritten as

$$
S(\boldsymbol{x}, z)=H(\boldsymbol{x}, z) \sum_{i=1}^{q} \frac{2(q-i)+1}{q^{2}} g_{[i]} .
$$

The summation multiplying the headcount ratio corresponds to an OWA function $A_{G}:[0,1]^{q} \longrightarrow[0,1]$ applied to the normalized poverty gaps,

$$
A_{G}\left(\boldsymbol{g}_{p}\right)=\sum_{i=1}^{q} w_{i} g_{[i]}, \quad w_{i}=\frac{2(q-i)+1}{q^{2}}, \quad i=1, \ldots, q .
$$

This OWA function $A_{G}$ satisfies continuity, idempotency (hence, compensativeness), symmetry, strict monotonicity, stability for translations, invariance for replications, and strict S-convexity. Moreover its core $\widehat{A}_{G}$ is idempotent, symmetric, strictly monotonic, and stable for translations. These properties can be regarded as basic properties of a poverty intensity index.

On the other hand, the anti-self-dual remainder $\widetilde{A}_{G}$ is symmetric, fulfills $\widetilde{A}_{G}\left(\boldsymbol{g}_{p}\right)=0$ if and only if $g_{[1]}=\cdots=g_{[q]}$, and is S-convex. Hence, $\widetilde{A}_{G}$ is a measure of inequality among the poor individuals. Since $\widetilde{A}_{G}$ is anti-self-dual, inequality among the poor does not change if we focus on poverty gaps, or on achievements as measured by $\boldsymbol{x}_{p} / z$. This component is also invariant if the units in which income is measured change. Moreover $\widetilde{A}_{G}$ is invariant for translations, thus it measures inequality from an absolute point of view and remains invariant if the gaps of all the poor are increased by the same amount.

In the particular case of the Sen index, we can establish that the core $\widehat{A}_{G}$ coincides with the aggregate income gap ratio,

$$
\widehat{A}_{G}\left(\boldsymbol{g}_{p}\right)=\frac{1}{q} \sum_{i=1}^{q} g_{[i]}=\mu\left(\boldsymbol{g}_{p}\right),
$$

the archetypical measure of poverty intensity. Moreover, the anti-self-dual remainder of the OWA function $A_{G}$ associated with the Sen index corresponds to 
the absolute Gini index of the normalized poverty gaps,

$$
\widetilde{A}_{G}\left(\boldsymbol{g}_{p}\right)=\mu\left(\boldsymbol{g}_{p}\right)-\sum_{i=1}^{q} \frac{2 i-1}{q^{2}} g_{[i]}=G_{A}\left(\boldsymbol{g}_{p}\right) .
$$

Finally, we conclude that the absolute Gini index $G_{A}\left(\boldsymbol{g}_{p}\right)$ of the normalized poverty gaps coincides with the absolute Gini index $G_{A}\left(\boldsymbol{x}_{p} / z\right)$ of the poor incomes normalized by the poverty line (see Aristondo et al. [1, Prop. 10]),

$$
G_{A}\left(\boldsymbol{g}_{p}\right)=G_{A}\left(\boldsymbol{x}_{p} / z\right) .
$$

\subsection{An application to classical inequality measures}

One of the most widely used inequality measure is the Gini index ([15]), based on the absolute values of all pairwise income differences. The Bonferroni index ([4]) measures inequality comparing the overall income mean with the income means of the poorest individuals in the population. The De Vergottini index ([7]) complements the information provided by the Bonferroni index since inequality is captured by comparing the overall income mean with the income means of the richest individuals in the population.

The welfare functions associated with the three classical inequality measures mentioned above are OWA functions. They can thus be analyzed in the framework of the dual decomposition presented in Subsection 2.3, as discussed in Aristondo et al. [2].

\subsubsection{The Gini index}

The Gini index is based on the average of the absolute differences between all possible pairs of incomes,

$$
G(\boldsymbol{x})=\frac{1}{2 n^{2} \mu(\boldsymbol{x})} \sum_{i=1}^{n} \sum_{j=1}^{n}\left|x_{i}-x_{j}\right|, \text { with } \boldsymbol{x} \neq \mathbf{0} .
$$

Mehran [21] highlights the implicit weighting scheme involved in the Gini index which assigns a particular weight to an individual according to his ranking in the income distribution,

$$
G(\boldsymbol{x})=1-\frac{1}{n^{2} \mu(\boldsymbol{x})} \sum_{i=1}^{n}(2 i-1) x_{[i]}, \text { with } \boldsymbol{x} \neq \mathbf{0} .
$$

When the Gini coefficient is multiplied by the mean income an absolute index, the absolute Gini inequality index is obtained,

$$
G_{A}(\boldsymbol{x})=\mu(\boldsymbol{x})-\frac{1}{n} \sum_{i=1}^{n} \frac{2 i-1}{n} x_{[i]} .
$$

The absolute Gini index $G_{A}$ is anti-self-dual (see Aristondo et al. [1, Prop. 2]),

$$
G_{A}(\mathbf{1}-\boldsymbol{x})=G_{A}(\boldsymbol{x}) .
$$


The Gini welfare function can be defined as

$$
W_{G}(\boldsymbol{x})=\mu(\boldsymbol{x})-G_{A}(\boldsymbol{x})=\frac{1}{n} \sum_{i=1}^{n} \frac{2 i-1}{n} x_{[i]} .
$$

The dual decomposition of the Gini welfare function is interesting (see Aristondo et al. [1, Prop. 13-14]): $\widehat{W}_{G}(\boldsymbol{x})=\mu(\boldsymbol{x})$ and $\widetilde{W}_{G}(\boldsymbol{x})=-G_{A}(\boldsymbol{x})$.

\subsubsection{The Bonferroni and De Vergottini indices}

The Bonferroni index [4] is based on the comparison between the partial means and the general mean of an income distribution. Let us denote by $m_{i}(\boldsymbol{x})$ the mean income of the $n-i+1$ individuals with lowest income, that is,

$$
m_{i}(\boldsymbol{x})=\frac{1}{n-i+1} \sum_{j=i}^{n} x_{[j]} .
$$

The Bonferroni index is defined as

$$
B(\boldsymbol{x})=\frac{1}{n \mu(\boldsymbol{x})} \sum_{i=1}^{n}\left(\mu(\boldsymbol{x})-m_{i}(\boldsymbol{x})\right), \text { with } \boldsymbol{x} \neq \mathbf{0} .
$$

In analogy with the Gini case, the absolute Bonferroni inequality index is defined as

$$
B_{A}(\boldsymbol{x})=\frac{1}{n} \sum_{i=1}^{n}\left(\mu(\boldsymbol{x})-m_{i}(\boldsymbol{x})\right)=\mu(\boldsymbol{x})-\frac{1}{n} \sum_{i=1}^{n} m_{i}(\boldsymbol{x}) .
$$

The Bonferroni welfare function is expressed by

$$
W_{B}(\boldsymbol{x})=\sum_{i=1}^{n} u_{i} x_{[i]}, \quad \text { with } \quad u_{i}=\sum_{j=n-i+1}^{n} 1 /(j n), \quad i=1, \ldots, n .
$$

The De Vergottini index ([7]) captures another aspect of the inequality. It compares the general mean of the income distribution with the partial means associated with the richest individuals in the popuation. If $M_{i}(\boldsymbol{x})$ denotes the mean income of the $i$ individuals with highest income, that is,

$$
M_{i}(\boldsymbol{x})=\frac{1}{i} \sum_{j=1}^{i} x_{[j]}
$$

then the De Vergottini index is

$$
V(\boldsymbol{x})=\frac{1}{n \mu(\boldsymbol{x})} \sum_{i=1}^{n}\left(M_{i}(\boldsymbol{x})-\mu(\boldsymbol{x})\right), \text { with } \boldsymbol{x} \neq \mathbf{0} .
$$

The De Vergottini index $V$ is also a compromise index in the sense that if multiplied by the mean, then the counterpart absolute index is obtained. 
The absolute De Vergottini inequality index is defined as

$$
V_{A}(\boldsymbol{x})=\frac{1}{n} \sum_{i=1}^{n}\left(M_{i}(\boldsymbol{x})-\mu(\boldsymbol{x})\right)=\frac{1}{n} \sum_{i=1}^{n} M_{i}(\boldsymbol{x})-\mu(\boldsymbol{x}) .
$$

The duality relation between the absolute Bonferroni and the absolute De Vergottini inequality indices is expressed by

$$
B_{A}(\mathbf{1}-\boldsymbol{x})=V_{A}(\boldsymbol{x}) \quad \text { and } \quad V_{A}(\mathbf{1}-\boldsymbol{x})=B_{A}(\boldsymbol{x}),
$$

for every $\boldsymbol{x} \in[0,1]^{n}$ (see Aristondo et al. [2, Prop. 15]).

The self-dual core and the anti-self-dual remainder of the Bonferroni welfare function are given by

$$
\widehat{W}_{B}(\boldsymbol{x})=\mu(\boldsymbol{x})-\frac{B_{A}(\boldsymbol{x})-V_{A}(\boldsymbol{x})}{2} \quad \text { and } \quad \widetilde{W}_{B}(\boldsymbol{x})=-\frac{B_{A}(\boldsymbol{x})+V_{A}(\boldsymbol{x})}{2} .
$$

We omit the analysis of the De Vergottini welfare function and its dual decomposition because of its complexity (see Aristondo et al. [2]).

\section{Concluding remarks}

Although aggregation functions are implicitly involved in welfare, social inequality, poverty, health, and quality of life measurement, the potential offered by the theory of aggregation functions does not emerge explicitly.

As briefly shown in Section 3, the dual decomposition of aggregation functions (García-Lapresta and Marques Pereira [14]) can provide interesting results in the field of welfare economics. For further details and references see GarcíaLapresta et al. [11] and Aristondo et al. [1, 2].

Other applications of the theory of aggregation functions to the field of welfare economics, in this case involving Choquet integration - a generalization of both weighted means and OWA functions, can be found in Bortot and Marques Pereira [5].

\section{Acknowledgments}

J.L. García-Lapresta gratefully acknowledges the funding support of the Spanish Ministerio de Economía y Competitividad (Project ECO2012-32178) and the Consejería de Educación de la Junta de Castilla y León (Project VA066U13).

\section{References}

[1] O. Aristondo, J.L. García-Lapresta, C. Lasso de la Vega, R.A. Marques Pereira, The Gini index, the dual decomposition of aggregation functions, and the consistent measurement of inequality, International Journal of Intelligent Systems 27 (2012) 132-152. 
[2] O. Aristondo, J.L. García-Lapresta, C. Lasso de la Vega, R.A. Marques Pereira, Classical inequality indices, welfare and illfare functions, and the dual decomposition, Fuzzy Sets and Systems 228 (2013) 114-136.

[3] G. Beliakov, A. Pradera, T. Calvo, Aggregation Functions: A Guide for Practitioners, Springer, Heidelberg, 2007.

[4] C. Bonferroni, Elementi di Statistica Generale, Libreria Seber, Firenze, 1930.

[5] S. Bortot, R.A. Marques Pereira, The binomial Gini inequality indices and the binomial decomposition of welfare functions, Fuzzy Sets and Systems 255 (2014) 92-114.

[6] T. Calvo, A. Kolesárova, M. Komorníková, R. Mesiar, Aggregation operators: Properties, classes and construction methods, in: T. Calvo, G. Mayor, R. Mesiar (Eds.), Aggregation Operators: New Trends and Applications, Physica-Verlag, Heidelberg, 2002, pp. 3-104.

[7] M. De Vergottini, Sugli indici di concentrazione, Statistica 10 (1940) 445454.

[8] D. Dubois, H. Prade, A review of fuzzy set aggregation connectives, Information Sciences 36 (1985) 85-121.

[9] J. Fodor, J.L. Marichal, M. Roubens, Characterization of the ordered weighted averaging operators, IEEE Transtactions on Fuzzy Systems 3 (1995) 236-240.

[10] J. Fodor, M. Roubens, Fuzzy Preference Modelling and Multicriteria Decision Support, Kluwer Academic Publishers, Dordrecht, 1994.

[11] J.L. García-Lapresta, C. Lasso de la Vega, R.A. Marques Pereira, A.M. Urrutia, A class of poverty measures induced by the dual decomposition of aggregation functions, International Journal of Uncertainty, Fuzziness and Knowledge-Based Systems 18 (2010) 493-511.

[12] J.L. García-Lapresta, B. Llamazares, Aggregation of fuzzy preferences: Some rules of the mean, Social Choice and Welfare 17 (2000) 673-690.

[13] J.L. García-Lapresta, R.A. Marques Pereira, Constructing reciprocal and stable aggregation operators, in: Proceedings of International Summer School on Aggregation Operators and their Applications, Alcalá de Henares, Spain (2003) 73-78.

[14] J.L. García-Lapresta, R.A. Marques Pereira, The self-dual core and the anti-self-dual remainder of an aggregation operator, Fuzzy Sets and Systems 159 (2008) 47-62.

[15] C. Gini, Variabilità e Mutabilità, Tipografia di Paolo Cuppini, Bologna, 1912. 
[16] M. Grabisch, J.L. Marichal, R. Mesiar, E. Pap, Aggregation Functions, Cambridge University Press, Cambridge, 2009.

[17] S. Jenkins, P. Lambert, Three 'I's of poverty curves and poverty dominance: TIPs for poverty analysis. Research on Economic Inequality 8 (1998) 39-56.

[18] A. Kolmogoroff, Sur la notion de la moyenne, Atti della R. Academia Nazionale del Lincei. Rendiconti della Classe di Scienze Fisiche, Mathematiche e Naturali (6) 12 (1930) 388-391.

[19] K.C. Maes, B. De Baets, Commutativity and self-duality: Two tales of one equation, International Journal of Approximate Reasoning 50 (2009) $189-199$.

[20] K.C. Maes, S. Saminger, B. De Baets, Representation and construction of self-dual aggregation operators, European Journal of Operational Research 177 (2007) 472-487.

[21] F. Mehran, Linear measures of income inequality, Econometrica 44 (1976) 805-809.

[22] M. Nagumo, Über eine Klasse der Mittelwerte, Japanese Journal of Mathematics 7 (1930) 71-79.

[23] A.K. Sen, Poverty: An ordinal approach to measurement, Econometrica 44 (1976) 219-231.

[24] W. Silvert, Symmetric summation: A class of operations on fuzzy sets, IEEE Transactions on Systems, Man, and Cybernetics 9 (1979) 657-659.

[25] R.R. Yager, Ordered weighted averaging operators in multicriteria decision making, IEEE Transactions on Systems, Man and Cybernetics 8 (1988) $183-190$. 